\title{
Modeling Multilook Magnitude and Phase in Extremely Heterogeneous Clutter
}

\author{
Gui Gao, Gongtao Shi, and Shilin Zhou \\ College of Electronic Science and Engineering, National University of Defense Technology, Changsha, \\ Hunan 410073, China \\ Correspondence should be addressed to Gui Gao; dellar@126.com
}

Received 30 December 2012; Accepted 18 January 2013

Academic Editor: Deren Li

Copyright (c) 2013 Gui Gao et al. This is an open access article distributed under the Creative Commons Attribution License, which permits unrestricted use, distribution, and reproduction in any medium, provided the original work is properly cited.

This paper proposes a joint distribution of magnitude and phase for multilook SAR interferogram in extremely heterogeneous clutter. The presented theoretical distribution, called simply here $\mathcal{J}^{2}$ distribution and derived from the multiplicative model and the known joint distribution of the homogeneous clutter, is shown to be able to model the extremely heterogeneous clutter areas. Moreover, we estimate distribution parameters by means of the well-known method-of-log-cumulants (MoLC). The experimental results applied on actual dual-channel SAR images prove the good performance of the proposed distribution.

\section{Introduction}

Ground moving target identification (GMTI) using synthetic aperture radar (SAR) has been a growing interest over the last couple of decades in many applications, such as military surveillance and reconnaissance of ground vehicles and civilian ship monitoring of harbor [1-3]. The recent works $[3,4]$ reported in this field show that the multilook interferogram, defined as the product of the first channel and the complex conjugate of the second, is an important tool for detecting moving targets. However, precise knowledge of the interferogram's phase and magnitude statistics, that is, the joint probability density function (PDF), is a major issue currently under study in the development of statistically based detector tests for distinguishing the moving targets from clutter [3-5].

Some investigations for statistical modeling of multilook SAR interferogram have been presented in the past, for example, [3-5]. Lee et al. [5] firstly proposed the joint distribution of interferometric magnitude and phase with the condition of a constant radar cross section (RCS) background based on the complex Wishart distribution, presented by Goodman
[6]. The analytical expression of this joint distribution is given as [5]

$$
\begin{aligned}
& p_{\xi, \psi}(\xi, \psi) \\
& \quad=\frac{2 n^{n+1} \xi^{n}}{\pi \Gamma(n)\left(1-\rho^{2}\right)} \exp \left(\frac{2 n \rho \xi \cos \psi}{1-\rho^{2}}\right) K_{n-1}\left(\frac{2 n \xi}{1-\rho^{2}}\right),
\end{aligned}
$$

where $\xi$ and $\psi$ are the normalized interferogram's magnitude and the multilook phase. $n$ represents number of looks, and $\rho$ indicates the magnitude of the complex correlation coefficient. $K_{n-1}(\cdot)$ is the second type modified Bessel function with order $(n-1) . \Gamma(\cdot)$ indicates the Gamma function. In the analysis of lot of literatures, it is shown [3-5] that the PDF shown in (1) is valid for modeling homogeneous areas, whereas it also tends to deviate strongly in most cases whose scenes contain heterogeneous or extremely heterogeneous regions.

Additionally, as the phase statistic is highly invariant against changes of the clutter type [3], the marginal PDFs of interferometric magnitude for heterogeneous and extremely heterogeneous regions are derived by Gierull [4]. Meanwhile, 
an original joint PDF of interferometric magnitude and phase for heterogeneous clutter is also given in afore-mentioned literature. Unfortunately, the joint PDFs of interferometric magnitude and phase for extremely heterogeneous regions are still a hard task by means of combining the marginal PDFs of magnitude and the ones of phase owing to that $\xi$ and $\psi$ are not statistically independent [3].

In this paper, our objective is to present a novel joint distribution of interferometric magnitude and phase for extremely heterogeneous clutter. We test the performance of the proposed distribution utilizing a representative dualchannel SAR image of urban area described as an extremely heterogeneous region.

\section{The Joint Distribution}

2.1. The Known Joint Distribution for Heterogeneous Regions. Considering an $n$-look interferogram $I_{n}$, it is the average of $n$ single-look interferograms. Assuming the energy of two channels is identical, it is well known that $I_{n}$ can be modeled by the multiplicative model as [4]

$$
I_{n}=A^{2} \xi e^{j \psi}
$$

where $A$ represents the backscattering RCS magnitude of each channel.

As analyzed by Frery et al. [7], the random variable $A$ obeys a reciprocal of the square root of a Gamma distribution to characterize highly heterogeneous situation, that is, $A \sim \Gamma^{-1 / 2}(-\alpha, \gamma)$. Supposing $W=A^{2}$, the PDF of $W$ is given by

$$
p_{W}(w)=\frac{\gamma^{-\alpha}}{\Gamma(-\alpha)} w^{\alpha-1} \exp \left(-\frac{\gamma}{w}\right), \quad-\alpha, \gamma>0,
$$

where $\alpha(-\alpha \in(0, \infty))$ is a shape parameter, which essentially reflects the degrees of homogeneity for processed areas. $\gamma$ is a scale parameter related to the mean energy of processed areas.

Therefore, the modified interferometric magnitude $\eta$ of heterogeneous clutter is given as $\eta=A^{2} \xi:=W \xi$ and the joint distribution of $\eta$ and $\psi$ can be expressed by

$$
p_{\eta, \psi}(\eta, \psi)=\int_{0}^{\infty} p_{W}(w) p(\eta \mid w, \psi) d w .
$$

Applying (1), we get the right hand-side of the integral shown in (4) as

$$
\begin{aligned}
p(\eta \mid w, \psi) & =\frac{1}{w} p\left(\frac{\eta}{w}, \psi\right) \\
= & \frac{2 n^{n+1} \eta^{n}}{\pi \Gamma(n)\left(1-\rho^{2}\right)}\left(\frac{1}{w}\right)^{n+1} \\
& \quad \times \exp \left(\frac{2 n \eta \rho \cos \psi}{1-\rho^{2}} \times \frac{1}{w}\right) K_{n-1}\left(\frac{2 n \eta}{1-\rho^{2}} \times \frac{1}{w}\right) .
\end{aligned}
$$

Combining (3) and (5) by (4), and utilizing the integral formula $\int_{0}^{\infty} x^{\mu-1} e^{-a x} K_{v}(b x) d x=\left(\sqrt{\pi}(2 b)^{v} /(a+b)^{\mu+v}\right)$.
$((\Gamma(\mu+v) \Gamma(\mu-v)) / \Gamma(\mu+1 / 2)){ }_{2} F_{1}(\mu+v, v+1 / 2 ; \mu+1 / 2 ;(a-$ $b) /(a+b))[8]$, the joint distribution of magnitude and phase in the heterogeneous clutter is finally derived as [4]

$$
\begin{aligned}
p_{\eta, \psi}(\eta, \psi) & \frac{(2 n)^{2 n} \gamma^{-\alpha}}{2 \sqrt{\pi}\left(1-\rho^{2}\right)^{-(n-\alpha)}} \cdot \frac{\Gamma(2 n-\alpha) \Gamma(-\alpha+2)}{\Gamma(n) \Gamma(-\alpha) \Gamma(n-\alpha+3 / 2)} \cdot \eta^{2 n-1} \\
& \cdot\left[f_{1}(\eta, \psi)\right]^{-(2 n-\alpha)} \\
& \cdot{ }_{2} F_{1}\left(2 n-\alpha, n-\frac{1}{2} ; n-\alpha+\frac{3}{2} ; f_{2}(\eta, \psi)\right),
\end{aligned}
$$

where ${ }_{2} F_{1}$ is the Gauss hypergeometric function and

$$
\begin{aligned}
& f_{1}(\eta, \psi)=\left(1-\rho^{2}\right) \gamma+2 n \eta(1-\rho \cos \psi), \\
& f_{2}(\eta, \psi)=\frac{\left(1-\rho^{2}\right) \gamma-2 n \eta(1+\rho \cos \psi)}{\left(1-\rho^{2}\right) \gamma+2 n \eta(1-\rho \cos \psi)} .
\end{aligned}
$$

2.2. The New Joint Distribution for Extremely Heterogeneous Regions. For extremely heterogeneous clutter like the urban areas, the histograms show the heavy trail. To solve this problem, Gierull [4] proposed a transformation of $\eta$ into $\varsigma=$ $\eta^{\delta}, \delta \in \mathbb{R}^{+}$, where the interferometric magnitude $\varsigma$ and phase of extremely heterogeneous clutter can be derived by (6) with Jacobian $\operatorname{det}|\mathbf{J}|=(1 / \delta) \eta^{1 / \delta^{-1}}$ to

$$
\begin{aligned}
p_{\varsigma, \psi}(\varsigma, \psi) & \\
= & \frac{(2 n)^{2 n} \gamma^{-\alpha}}{2 \sqrt{\pi} \delta\left(1-\rho^{2}\right)^{-(n-\alpha)}} \cdot \frac{\Gamma(2 n-\alpha) \Gamma(-\alpha+2)}{\Gamma(n) \Gamma(-\alpha) \Gamma(n-\alpha+3 / 2)} \\
& \cdot \varsigma^{(2 n / \delta)-1}\left[f_{1}^{\prime}(\varsigma, \psi)\right]^{-(2 n-\alpha)} \\
& \times{ }_{2} F_{1}\left(2 n-\alpha, n-\frac{1}{2} ; n-\alpha+\frac{3}{2} ; f_{2}^{\prime}(\varsigma, \psi)\right),
\end{aligned}
$$

where $f_{1}^{\prime}(\varsigma, \psi)$ and $f_{2}^{\prime}(\varsigma, \psi)$ are the following functions:

$$
\begin{aligned}
& f_{1}^{\prime}(\varsigma, \psi)=\left(1-\rho^{2}\right) \gamma+2 n \varsigma^{1 / \delta}(1-\rho \cos \psi), \\
& f_{2}^{\prime}(\varsigma, \psi)=\frac{\left(1-\rho^{2}\right) \gamma-2 n \varsigma^{1 / \delta}(1+\rho \cos \psi)}{\left(1-\rho^{2}\right) \gamma+2 n \varsigma^{1 / \delta}(1-\rho \cos \psi)} .
\end{aligned}
$$

Hereafter (8) is denoted simply by $\mathscr{J}^{2}$. The marginal PDF of interferometric magnitude $\varsigma$ is further given by integrating (8) with respect to the phase $\psi$ as

$$
\begin{aligned}
p_{\varsigma}(\varsigma)= & \left(\frac{2 n}{\gamma(1+\rho)}\right)^{n} \cdot \frac{\Gamma(n-\alpha)}{\delta \Gamma(n) \Gamma(-\alpha)} \\
& \cdot \frac{\varsigma^{(n / \delta)^{-1}}}{\left(1+(2 n / \gamma(1+\rho)) \varsigma^{1 / \delta}\right)^{n-\alpha}}, \quad \rho,-\alpha, \gamma, n, \delta, \varsigma>0 .
\end{aligned}
$$




$$
\begin{array}{cc}
\delta \rightarrow 1, & -\alpha, \gamma \rightarrow \infty, \\
-\alpha, \gamma>0 & (-\alpha / \gamma) \rightarrow 1 \\
p_{\zeta, \psi}(\varsigma, \psi)->p_{\eta, \psi}(\eta, \psi) & \begin{array}{c}
(-\alpha, \psi \\
-d \vec{D}
\end{array} \quad \vec{D}
\end{array}
$$

FIGURE 1: The relationship of the joint distributions.

2.3. Relationship between Distributions. The relations of the aforementioned joint distributions are summarized in Figure 1 , where the symbol $\vec{D}$ denotes the convergence in distribution. It is clear from the derivations of (5) and (8) that the $g^{2}$ distribution converges to the $p_{\eta, \psi}(\eta, \psi)$ when $-\alpha, \gamma>$ $0, \delta \rightarrow 1$. Similarly, $p_{\eta, \psi}(\eta, \psi)$ also converges to $p_{\xi, \psi}(\xi, \psi)$ under the condition that $-\alpha, \gamma \rightarrow \infty,(-\alpha / \gamma) \rightarrow 1$. The properties stated in Figure 1 show that either homogeneous, heterogeneous, or extremely heterogeneous interferogram's magnitude and phase statistics can be treated as the outcome of the proposed $\mathscr{J}^{2}$ distribution.

\section{Parameter Estimations}

The MoLC, which relies on the Mellin transform [9], is a more feasible parametric PDF estimation technique for distributions defined on $[0,+\infty)$. Given $p$ as a function defined over $\mathbb{R}^{+}$, the Mellin transform of $p$ is defined as

$$
\mathscr{M}[p(x)](s)=\int_{0}^{\infty} x^{s-1} p(x) \mathrm{d} x .
$$

Thus, the second-kind first characteristic function and the second-kind second characteristic function are given, respectively, by

$$
\begin{gathered}
\phi(s)=\mathscr{M}[p(x)](s)=\int_{0}^{\infty} x^{s-1} p(x) \mathrm{d} x, \\
\Phi(s)=\ln \phi(s) .
\end{gathered}
$$

The estimate $\hat{\rho}$ of parameter $\rho$ has been obtained by Abdelfattah and Nicolas; the details can be found in [10]. However, here, we are interested in estimates of the parameters $\alpha, \gamma, n$, and $\delta$ in the $\mathcal{J}^{2}$ distribution. To make the deriving process be simplified, we notice that the parameters of the $\mathscr{J}^{2}$ distribution and the corresponding marginal PDF of magnitude given by (10) are identical. Motivated by this property, by plugging (10) into (12), one gets

$$
\begin{aligned}
\phi(s)= & \left(\frac{\gamma(1+\rho)}{2 n}\right)^{\delta(s-1)} \\
& \cdot \frac{\Gamma(n+\delta(s-1)) \Gamma(-\alpha-\delta(s-1))}{\Gamma(n) \Gamma(-\alpha)}, \\
\Phi(s)= & \delta(s-1) \ln \left(\frac{\gamma(1+\rho)}{2 n}\right)+\ln \Gamma(n+\delta(s-1)) \\
& +\ln \Gamma(-\alpha-\delta(s-1))-\ln \left(\frac{\Gamma(n)}{\Gamma(-\alpha)}\right) .
\end{aligned}
$$

The $k$ th-order derivative of $\Phi(s)$ at $s=1$ is the $k$ thorder second-kind cumulant also named "log-cumulant."

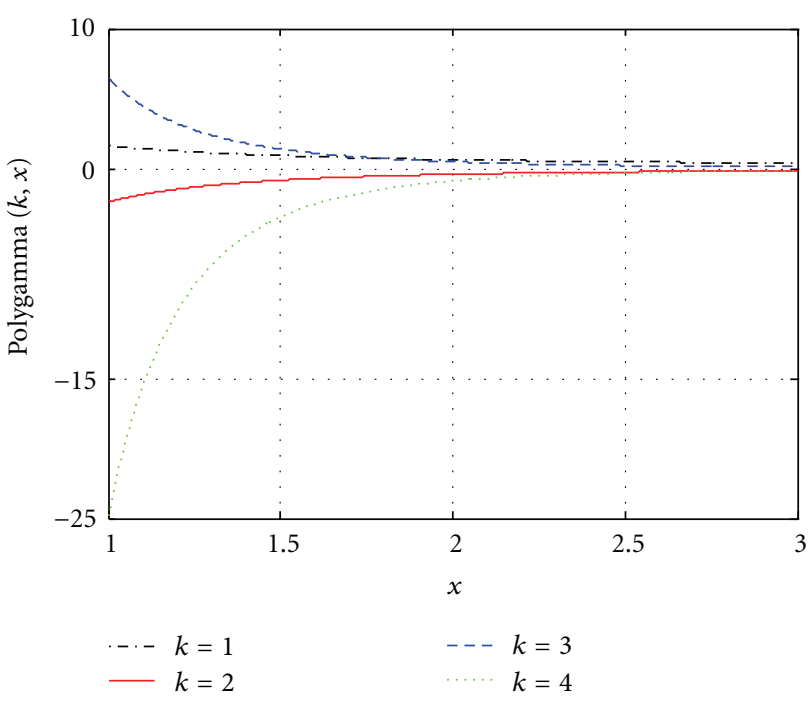

Figure 2: The $\Psi(k, \cdot)$ function.

Consequently, the $k$ th-order log-cumulants corresponding to the $\mathscr{J}^{2}$ distribution are

$$
\begin{gathered}
\tilde{c}_{1}=\delta\left[\ln \left(\frac{\gamma(1+\hat{\rho})}{2 n}\right)+\Psi(n)-\Psi(-\alpha)\right], \\
\widetilde{c}_{k}=\delta^{k}\left[\Psi(k-1, n)+(-1)^{k} \Psi(k-1,-\alpha)\right], \quad k \geq 2,
\end{gathered}
$$

where $\Psi(\cdot)$ represents the digamma function and $\Psi(k, \cdot)$ is the $k$ th-order polygamma function. Additionally, the logcumulants $\widetilde{c}_{k}$ can be directly estimated by $N$ samples $x_{i}$ as follows:

$$
\begin{gathered}
\widehat{\widetilde{c}}_{1}=\frac{1}{N} \sum_{i=1}^{N}\left[\ln \left(x_{i}\right)\right], \\
\widehat{\widetilde{c}}_{k}=\frac{1}{N} \sum_{i=1}^{N}\left[\left(\ln \left(x_{i}\right)-\widehat{\widetilde{c}}_{1}\right)^{k}\right], \quad k \geq 2 .
\end{gathered}
$$

The $\Psi(k, \cdot)$ functions with varying $k$ are shown in Figure 2 Thus (15) is continuous and strictly monotonically for each parameter of $\delta, n$, and $\alpha$. To obtain the numerical solution quickly and simply, we set $k$ as an even (i.e., letting $k=2$, $k=4$ and $k=6$ in (15), resp.). On the other hand, we stress that (15) does not contain $\gamma$, thus allowing us to split the nonlinear solution procedure into two distinct stages. First, the estimates $\widehat{\delta}, \widehat{n}$, and $\widehat{\alpha}$ of the parameters $\delta, n$, and $\alpha$ are accquired by solving (15) and (16), that is,

$$
\begin{aligned}
& \widehat{\delta}^{2}[\Psi(1, \widehat{n})+\Psi(1,-\widehat{\alpha})]=\frac{1}{N} \sum_{i=1}^{N}\left[\left(\ln \left(x_{i}\right)-\widehat{\widetilde{\widetilde{c}}}_{1}\right)^{2}\right], \\
& \widehat{\delta}^{4}[\Psi(3, \widehat{n})+\Psi(3,-\widehat{\alpha})]=\frac{1}{N} \sum_{i=1}^{N}\left[\left(\ln \left(x_{i}\right)-\widehat{\widetilde{c}}_{1}\right)^{4}\right], \\
& \widehat{\delta}^{6}[\Psi(5, \widehat{n})+\Psi(5,-\widehat{\alpha})]=\frac{1}{N} \sum_{i=1}^{N}\left[\left(\ln \left(x_{i}\right)-\widehat{\widetilde{c}}_{1}\right)^{6}\right] .
\end{aligned}
$$




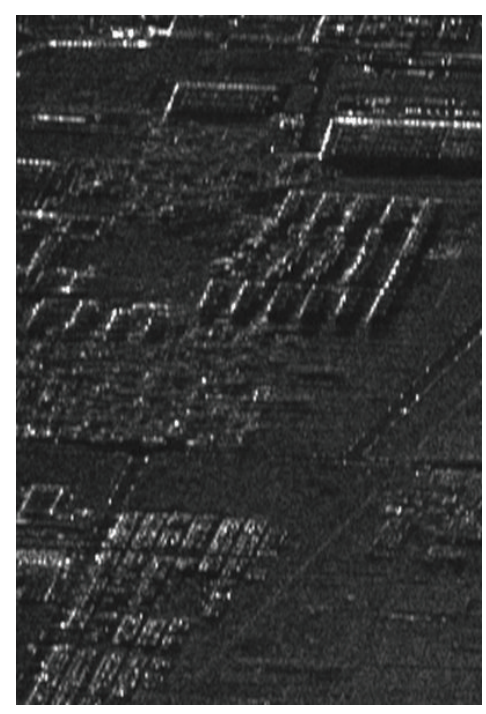

FIgURE 3: The tested SAR data of urban.

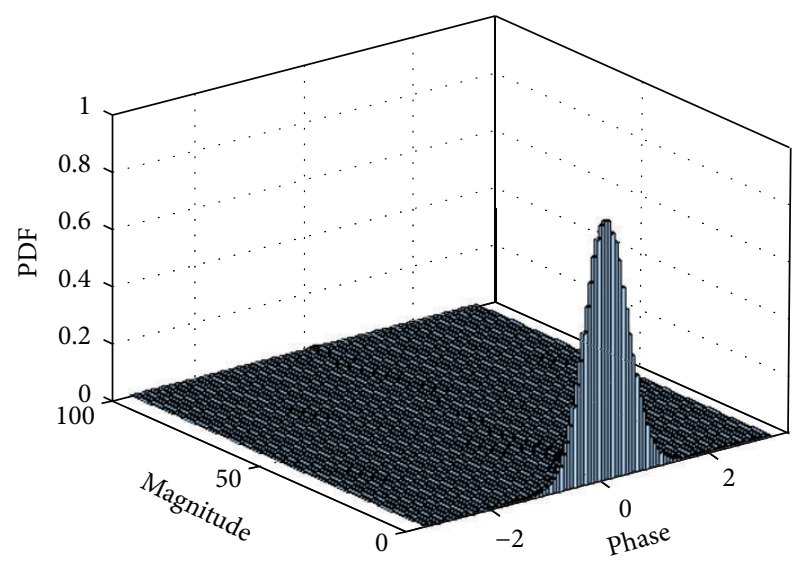

FIgURE 4: The three-dimension histogram of the tested urban area.

Second, via (14) and (16), the estimate $\widehat{\gamma}$ of the parameter $\gamma$ is

$$
\widehat{\gamma}=2 \widehat{n} \exp \frac{\left((1 / N \widehat{\delta}) \sum_{i=1}^{N}\left[\ln \left(x_{i}\right)\right]-\Psi(\widehat{n})+\Psi(-\widehat{\alpha})\right)}{(1+\widehat{\rho})} .
$$

\section{Experimental Analysis}

In this section, we present simulation results obtained by measured SAR data using the proposed $\mathscr{J}^{2}$ distribution. Especially, we provide the fitting performance of urban area indicated as extremely heterogeneous clutter to support the prior theoretical analysis that the $\mathscr{J}^{2}$ distribution is able to model the clutter areas with widely varying degrees of homogeneity.

As a representative example, the test dual-channel SAR data of urban used in this study were acquired by an airborne SAR system of China in Beijing operated in X band and $\mathrm{HH}$ polarization, with the spatial resolution $10 \mathrm{~m} \times 2 \mathrm{~m}$ (azimuth

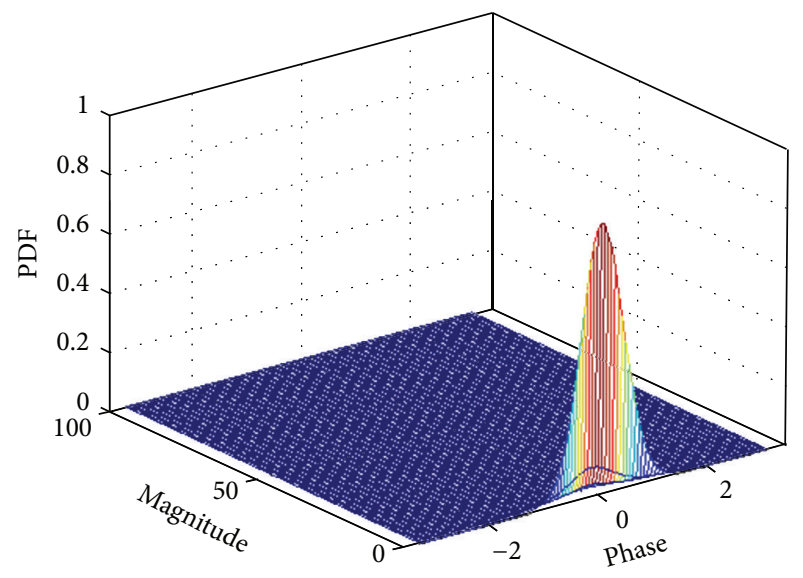

Figure 5: The estimated PDF of the previous area.

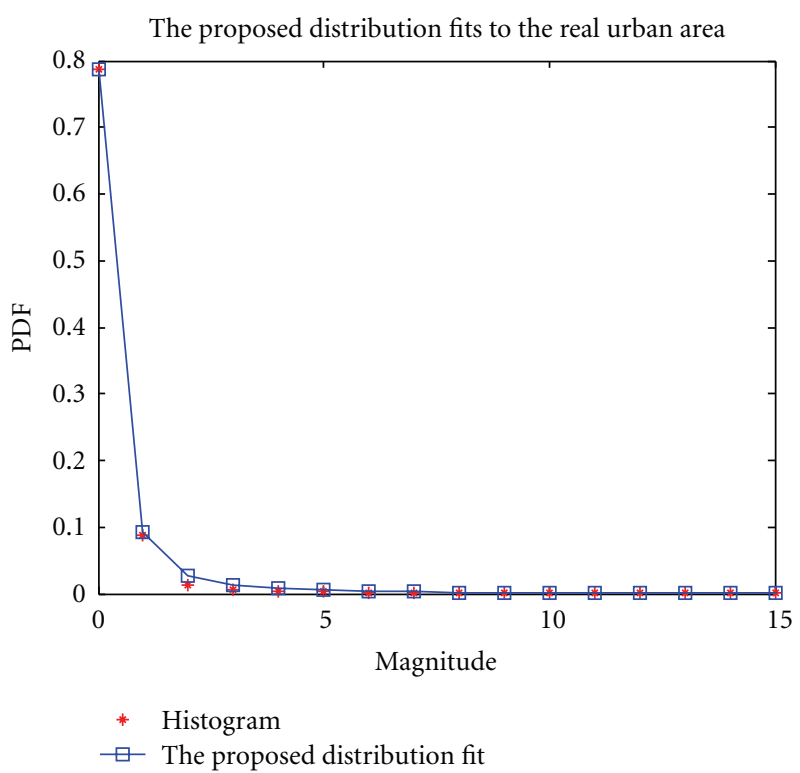

FIGURE 6: Plots of fitting results by limiting $\psi=0$

$\times$ range); see Figure 3 . The three-dimension histogram of the urban interferogram is shown in Figure 4.

We apply the proposed parametric estimation algorithm in Section 3 to the above-mentioned urban area. The results of parametric estimation of the corresponding $\mathscr{J}^{2}$ distribution are listed in Table 1. Based on these estimated parameters, the fitting histogram is given in Figure 5. It is clearly seen that the $\mathscr{J}^{2}$ distribution performs very well in fitting the histogram of the urban area from the viewpoint of the visual comparison between the histogram and the estimated PDF.

Furthermore, in order to assess the effectiveness of the presented $\mathscr{J}^{2}$ distribution, we test the developed model on the previous urban area by limiting various phase $\psi$ values. Figure 6 yields the fitting result with the condition of $\psi=0$. It is easy to observe that the $\mathcal{J}^{2}$ distribution agrees well with the given urban area which implies the wide modeling ability of the proposed distribution, as expected. 
TABLE 1: Parameter estimations of noted clutter areas in Figure 3.

\begin{tabular}{ccc}
\hline & $\widehat{\rho}$ & 0.7659 \\
$\mathscr{J}^{2}$ & $\widehat{n}$ & 0.9107 \\
& $\widehat{\alpha}$ & -0.9108 \\
& $\widehat{\gamma}$ & 0.1404 \\
& $\widehat{\delta}$ & 0.9897 \\
\hline
\end{tabular}

\section{Conclusion}

In this paper, we have developed a joint distribution of magnitude and phase for multilook SAR interferogram in extremely heterogeneous clutter. We also provide the corresponding parameter estimation technique based on the MoLC. The theoretical analysis and experimental results of measured multilook SAR data both have shown the good performance modeling extremely heterogeneous clutter. Since either homogeneous, heterogeneous, or extremely heterogeneous interferogram's magnitude and phase statistics can be treated as the outcome of the proposed $\mathscr{J}^{2}$ distribution (as Figure 1 stated), the presented distribution turns out to be suitable for the clutter with widely varying degrees of homogeneity.

\section{Acknowledgment}

The author would like to acknowledge the National Natural Science Foundation of China for the support under Grant 41171316.

\section{References}

[1] D. Cerutti-Maori, J. Klare, A. R. Brenner, and J. H. G. Ender, "Wide-area traffic monitoring with the SAR/GMTI system PAMIR," IEEE Transactions on Geoscience and Remote Sensing, vol. 46, no. 10, pp. 3019-3030, 2008.

[2] E. Chapin and C. W. Chen, "Along-track interferometry for ground moving target indication," IEEE Aerospace and Electronic Systems Magazine, vol. 23, no. 6, pp. 19-24, 2008.

[3] I. C. Sikaneta, Detection of ground moving objects with synthetic aperture radar [Ph.D. thesis], University of Ottawa, 2002.

[4] C. H. Gierull, "Statistical analysis of multilook SAR interferograms for CFAR detection of ground moving targets," IEEE Transactions on Geoscience and Remote Sensing, vol. 42, no. 4, pp. 691-701, 2004.

[5] J. S. Lee, K. W. Hoppel, S. A. Mango, and A. R. Miller, "Intensity and phase statistics of multilook polarimetric and interferometric SAR imagery," IEEE Transactions on Geoscience and Remote Sensing, vol. 32, no. 5, pp. 1017-1028, 1994.

[6] N. R. Goodman, "Statistical analysis based on a certain multivariate complex gaussian distribution (an introduction)," The Annals of Mathematical Statistics, vol. 34, no. 1, pp. 152-177, 1963.

[7] A. C. Frery, H. J. Muller, C. C. F. Yanasse, and S. J. S. Sant'Anna, "A model for extremely heterogeneous clutter," IEEE Transactions on Geoscience and Remote Sensing, vol. 35, no. 3, pp. 648-659, 1997.

[8] S. I. Gradshteyn and I. M. Ryzhik, Table of Integrals, Series, and Products, Academic Press, San Diego, Calif, USA, 7th edition, 2007.
[9] J. M. Nicolas, "Introduction to second kind statistic: application of log-moments and log-cumulants to SAR image law analysis," Traitement du Signal, vol. 19, no. 3, pp. 139-167, 2002.

[10] R. Abdelfattah and J. M. Nicolas, "Interferometric SAR coherence magnitude estimation using second kind statistics," IEEE Transactions on Geoscience and Remote Sensing, vol. 44, no. 7, pp. 1942-1953, 2006. 

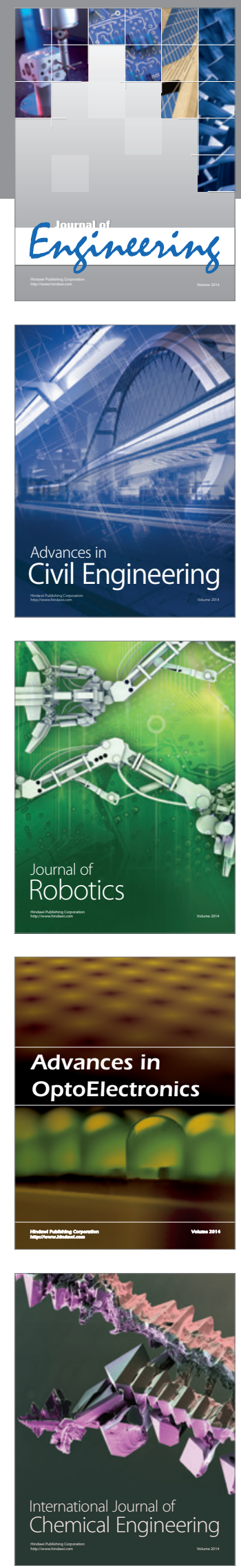

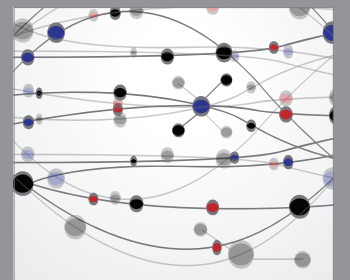

The Scientific World Journal
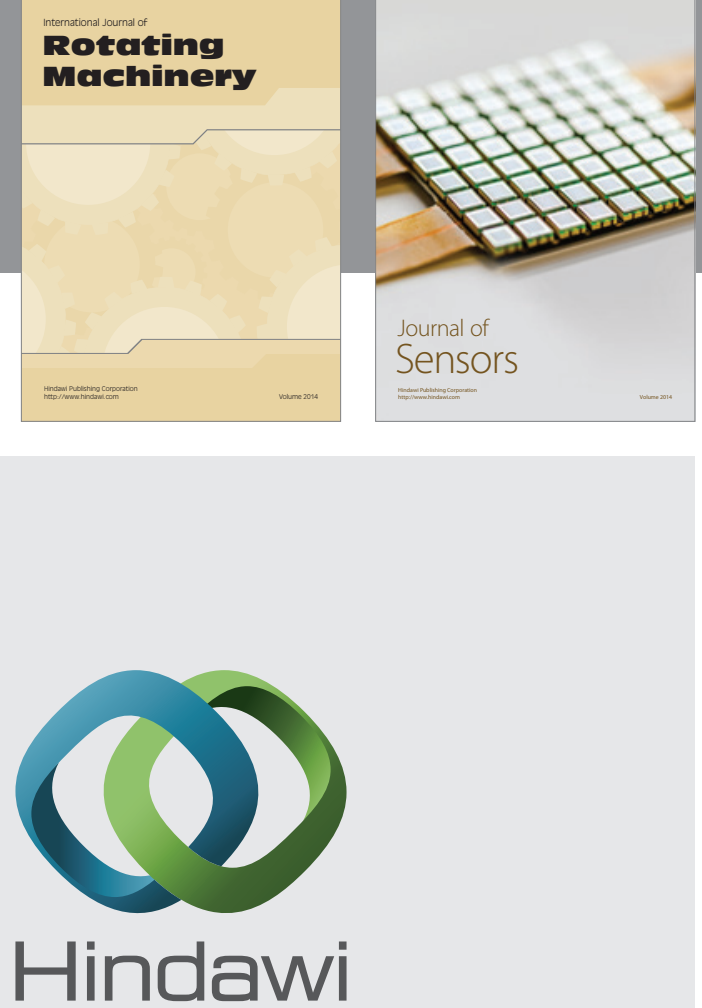

Submit your manuscripts at http://www.hindawi.com
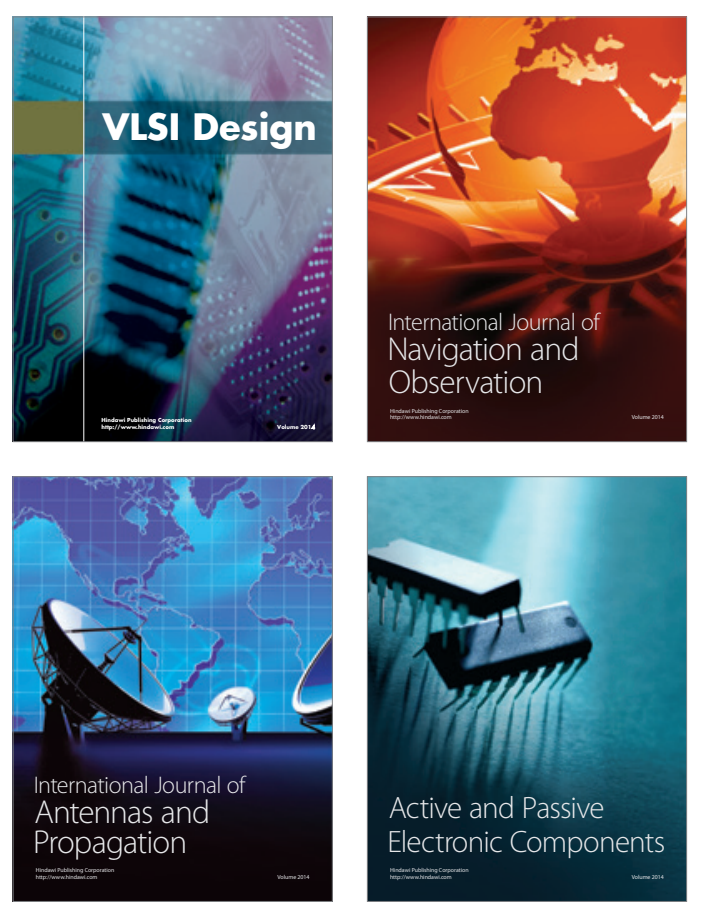
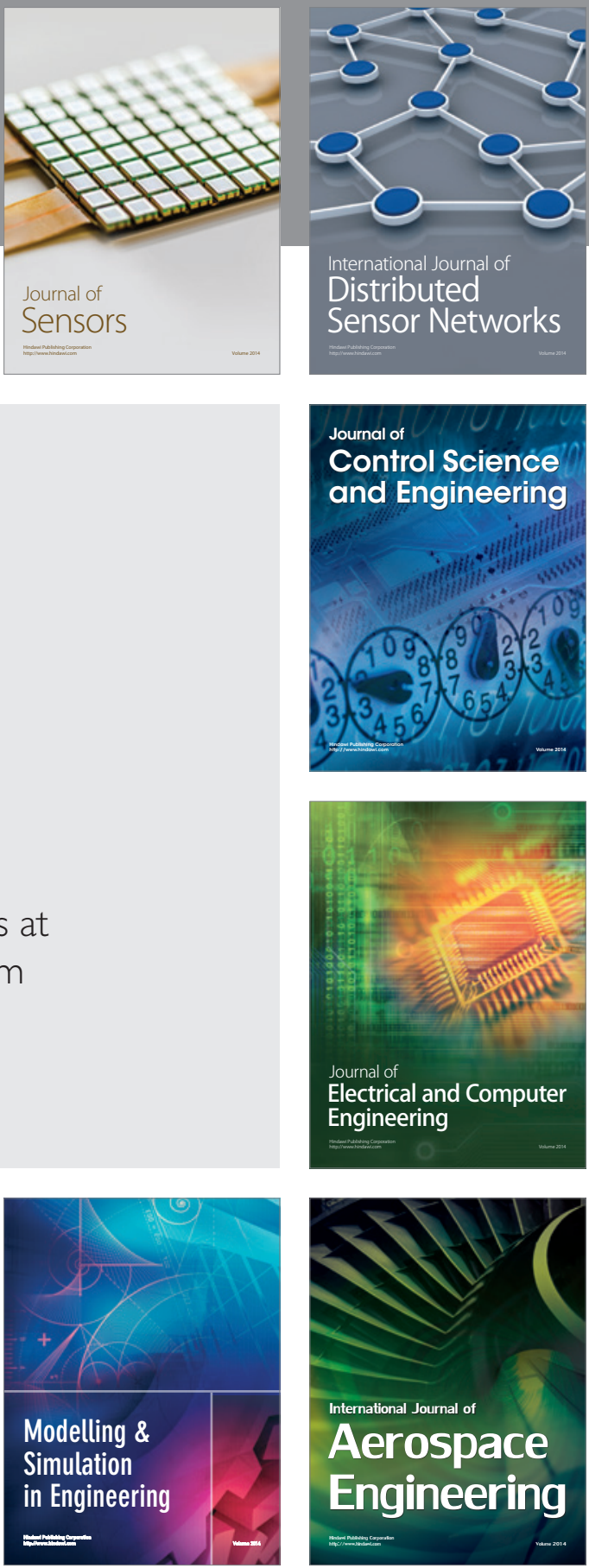

Journal of

Control Science

and Engineering
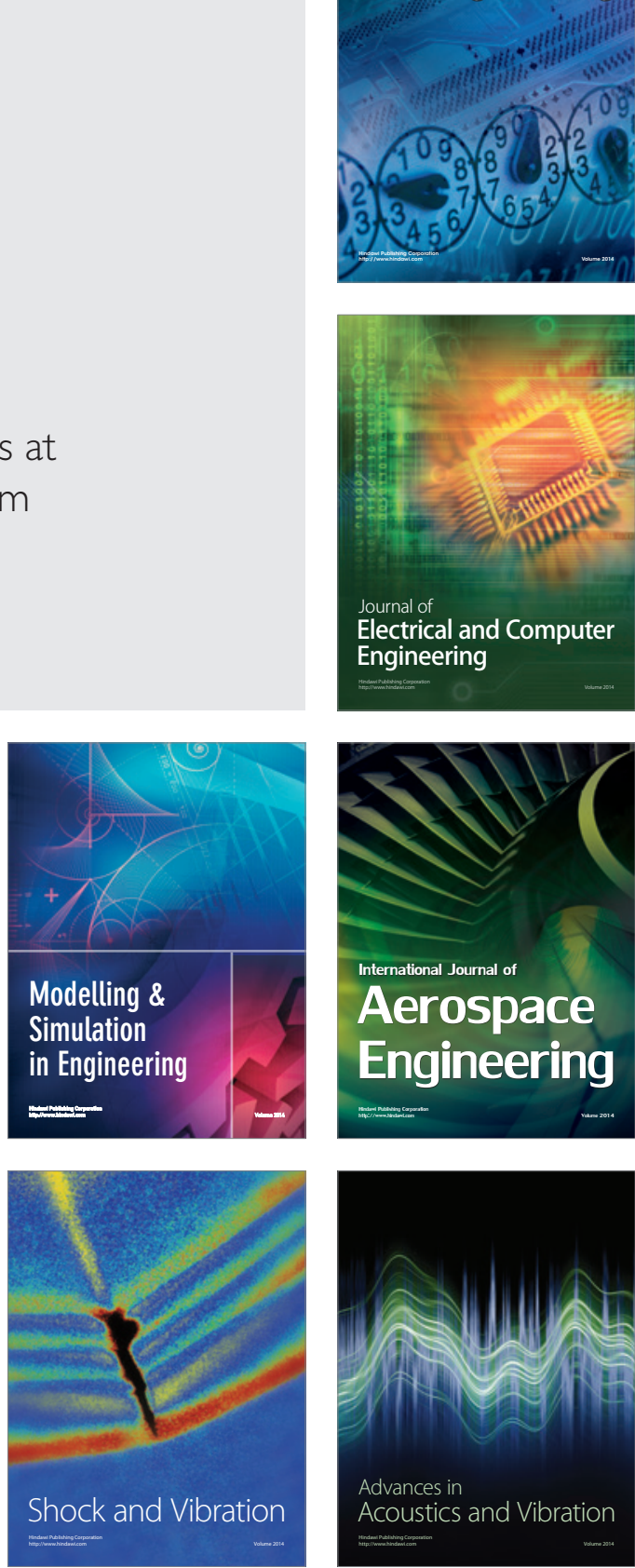